\section{Zhu ${ }^{1 *}$ and B.S. Nikolajczyk ${ }^{1,2}$}

${ }^{1}$ Department of Microbiology, Boston University School of Medicine, Boston, MA, USA; and ${ }^{2}$ Department of Medicine, Boston University School of Medicine, Boston, MA, USA;

*corresponding author, minzhu@bu.edu

J Dent Res 93(4):346-352, 2014

\section{Immune Cells Link Obesity- associated Type 2 Diabetes and Periodontitis}

\section{INTRODUCTION}

O besity prevalence has dramatically increased over the past 2 decades such that over 500 million adults are now categorized as obese [body mass index (BMI) $\geq 30.0 \mathrm{~kg} / \mathrm{m}^{2}$ ] (Swinburn et al., 2011). Obese individuals have a more than ten-fold risk of developing type 2 diabetes (T2D) compared with normal-weight individuals (Kopelman, 2007), although the appreciation of the subset of obese individuals who remain metabolically healthy (the metabolically healthy obese [MHO]; Fig. 1A) is growing (Denis and Obin, 2013). Classification of metabolic status in obese individuals is important for the clinician to interpret accumulating evidence of an association between non-diabetic obesity and periodontitis (Suvan et al., 2011), and the increased prevalence, progression, and severity of periodontitis in obese individuals compared with lean individuals (Gorman et al., 2012; Jimenez et al., 2012). This newly appreciated relationship between obesity and periodontitis is consistent with the well-established paradigm of a bi-directional relationship between obesity-associated T2D and periodontitis, including work showing that T2D increases susceptibility to periodontitis and that periodontal inflammation negatively affects glycemic control (Preshaw et al., 2012). Because obesity and T2D are highly interrelated, and both contribute to the development and progression of periodontal diseases, it is extremely challenging to delineate causal relationships among obesity, T2D, and periodontitis through clinical studies (Fig. 1B). Evidence from the clinic and experiments in model systems (in vivo and in vitro) show that multiple factors contribute to the pathogenesis of periodontitis in both obesity and obesity-associated T2D. Herein we highlight immune system changes that drive inflammation in obesity, obesityassociated T2D, and periodontitis and may mechanistically explain the clinical associations among these diseases, and may justify sub-classification of obese animals and people into MHO and metabolically unhealthy in terms of both experimental outcomes and treatment options.

\section{IMMUNE RESPONSES IN OBESITY AND OBESITY-ASSOCIATED T2D}

In contrast to $\mathrm{MHO}$, which is generally characterized as non-inflammatory (Denis and Obin, 2013), obesity-associated T2D is characterized by chronic low-grade inflammation. Most animal models of obesity have metabolic disease, including insulin resistance. Thus the "MHO" phenotype for animals occurs only under artificial conditions and is a relatively common outcome in mice that lack immunity-related genes. Regardless, the inflammatory nature of obesity-associated T2D raises the possibility that inflammation combines with obesity to promote T2D. Multiple studies have investigated the relationship between immunological and metabolic processes to explore the pathogenesis of obesity-associated T2D. One focus of this work is the dynamic 
nature of immune cell activation and tissue infiltration in obesity, with growing emphasis on pro-inflammatory functions of adipose-tissue-associated and recirculating cells of the immune system.

Adipose tissue cells, or adipocytes, manage lipid availability and secrete hormones (adipokines), including antiinflammatory adiponectin and pro-inflammatory leptin, to maintain metabolic homeostasis. Adipose tissue macrophages reside adjacent to adipocytes and exhibit an anti-inflammatory phenotype that maintains a non-inflammatory state in lean and perhaps $\mathrm{MHO}$ individuals (Lumeng et al., 2007; Chawla et al., 2011; Denis and Obin, 2013). Adipose tissue homeostasis is further reinforced by a unique population of $\mathrm{CD}^{+}$regulatory $\mathrm{T}$ cells (Tregs), which function largely as negative regulators of adipose tissue inflammation and systemic metabolic imbalance (Feuerer et al., 2009).

As obesity progresses and adipose tissue expands, adipocytes secrete less adiponectin and more leptin and chemokines, and adipose-associated immune cells change in parallel. It is not clear whether the original adipose-tissue-resident immune cells become directly activated in response to obesity; however, evidence indicates that circulating immune cells infiltrate the expanding adipose tissue in response to high-fat feeding (Oh et al., 2012). Although the exact order of immune cell infiltration into the expanding adipose tissue remains controversial, increased numbers of both neutrophils and macrophages appear early in disease pathogenesis (Elgazar-Carmon et al., 2008; Oh et al., 2012). B-cell infiltration followed by T cells (Duffaut et al., 2009; Winer et al., 2011) is also essential to obesity-associated inflammation. Although the proposed role of B-cell antibodies in inflammatory obesity remains controversial (Winer et al., 2011; DeFuria et al., 2013), B cells likely function at least in part through an ability to secrete pro-inflammatory cytokines and to regulate T-cell and macrophage-mediated inflammation (Winer et al., 2011; DeFuria et al., 2013). Infiltrating $\mathrm{CD} 8^{+} \mathrm{T}$ cells similarly regulate adipose tissue inflammation, as evidenced by demonstrations that obesity-associated $\mathrm{CD} 8^{+} \mathrm{T}$ cells secrete IFN- $\gamma$, an important pro-inflammatory cytokine for macrophage activation and obesity-induced inflammation (Rocha et al., 2008; Nishimura et al., 2009). $\mathrm{CD} 8^{+} \mathrm{T}$-cell deletion results in a relative lack of inflammatory macrophages in obese adipose tissue and, importantly, systemic insulin sensitivity (Nishimura et al., 2009). Finally, in contrast to the decreased numbers and percentages of Tregs in adipose tissue and in circulation during obesity (Feuerer et al., 2009; DeFuria et al., 2013), CD4 $4^{+}$Th1 cells expand and further contribute to overall IFN- $\gamma$ amounts (Rocha et al., 2008; Winer
B

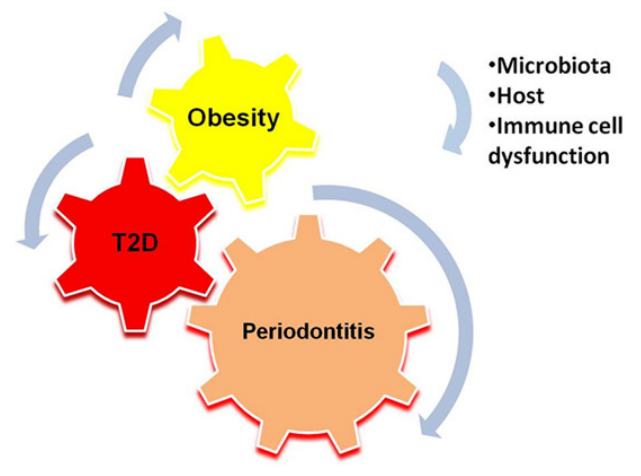

-Host

-Immune cell dysfunction

Figure 1. The relationships among obesity, type 2 diabetes (T2D), and periodontitis. (A) Obesity and T2D are highly interrelated in humans. Under conditions of calorie excess (dietinduced obesity), many people develop metabolically abnormal obesity (MAO), which includes individuals with obesity-associated T2D. However, some with T2D are not obese, and this is more prevalent in some genetic backgrounds. There are also individuals labeled 'metabolically healthy obese' (MHO) who are relatively protected from obese-associated seen in wild-type mouse models, since mice usually develop obesity and metabolic bi-directional relationship between $\mathrm{T} 2 \mathrm{D}$ and periodontitis are well-appreciated. It has been recently established that there is an association between obesity and periodontitis. Analysis of a influencing the other 2. The altered microbiota and dysregulated host response (including (arrows) of these disease gears. Studie often fail to identify obese individuals adequately as $\mathrm{MHO}$ or as metabolically unhealthy; thus the location of $\mathrm{MHO}$ in this panel is unknown.

et al., 2009; Nikolajczyk et al., 2012). Pro-inflammatory CD4 Th17s also accumulate in obese adipose tissue (Winer et al., 2009; DeFuria et al., 2013). Overall, the shift from dominance of anti-inflammatory Tregs to pro-inflammatory Th1/Th17s (Feuerer et al., 2009; Winer et al., 2009; Nikolajczyk et al., 2012) reinforces macrophage and B-cell pro-inflammatory cytokine profiles in obesity-associated T2D.

$\mathrm{B}$ cells and $\mathrm{T}$ cells play critical roles in obesity-associated inflammation and insulin resistance, but myeloid cells eventually dominate obese adipose tissue inflammation through relatively well-understood mechanisms. Saturated free fatty acids and/or hyperglycemia activates macrophages, thus triggering a cytokine-driven inflammatory cascade and insulin resistance, primarily via TNF- $\alpha$, IL-6, and IL-1 $\beta$, among others (Saberi et al., 2009; Chawla et al., 2011). Related work on obesityassociated dendritic cells (DCs), a macrophage cousin, shows that DCs contribute to inflammatory obesity by regulating a pro-inflammatory CD4 $4^{+} \mathrm{T}$-cell response (Bertola et al., 2012). DCs also initiate and regulate macrophage adipose tissue recruitment in obesity to fuel the feed-forward inflammatory loop (Stefanovic-Racic et al., 2012). Overall, over-nutrition and the resultant obesity (often confounded by metabolic imbalance) change both immune cell trafficking and function, and the vast majority of animal studies show that loss of pro-inflammatory immune cell function protects against obesity and/or obesityassociated insulin resistance. 


\section{IMMUNE RESPONSES IN PERIODONTITIS}

If obesity and/or obesity-associated T2D (obesity/T2D) potentiates periodontitis by priming the immune system, one might expect similar alterations in innate and adaptive immune cell function in the 2 diseases. Although the temporal order of immune cell infiltration and inflammation in chronically infected gingiva may differ from the order described above for the expanding adipose tissue in inflammatory obesity/T2D, many studies highlight the parallels between pro-inflammatory changes in individuals with periodontitis and obese individuals with T2D. Although many periodontitis studies have not reported the BMI or metabolic health status of human participants, most murine periodontitis studies have been completed on chow-fed mice, which (excepting select genotypes) are generally lean. For clarity, we have mentioned the obesity status of the individuals (when known) in the periodontitis studies highlighted below. However, very few studies have directly compared mechanisms of periodontitis in lean/non-diabetic and obese/non-diabetic individuals.

Just as adipocytes are the dominant cell type in lean and obese adipose tissue, gingival epithelial cells and fibroblasts are major cell types of gingival tissue that help maintain homeostasis. In healthy gingiva, epithelial cells and fibroblasts mediate a "simmering" innate immune response and direct gingival trafficking of more traditional immune system cells (Bosshardt and Lang, 2005). Gingival trafficking coordinates movement of neutrophils, among other cell types, which migrate through the junctional epithelium and into the gingival crevice to form a barrier against plaque biofilm in healthy gingiva. Although neutrophils also phagocytose and kill potentially pathogenic microorganisms, timely clearance of apoptotic neutrophils by macrophages (Hajishengallis and Hajishengallis, 2014) also links innate immune mechanisms to oral health.

Periodontitis in lean individuals is characterized by multiple changes in gingival innate immunity. Gingival epithelial cells and fibroblasts respond to pathogen LPS by the transient expression of cytokines such as TNF- $\alpha$ and IL-1 $\beta$; thus these cells likely play an active role in the initiation and maintenance of gingival inflammation (Miyauchi et al., 2001). In addition, neutrophils exhibit pro-survival and hyper-responsive phenotypes that extend neutrophil life and promote excessive production of reactive oxygen species (Dias et al., 2011; Lakschevitz et al., 2013), which further contribute to periodontitis pathogenesis. Interestingly, early neutrophil function in periodontitis is similar to the early contribution of neutrophils to obesity-associated adipose tissue inflammation, despite the sterile nature of the latter process. Periodontitis is also characterized by monocyte infiltration. The infiltrating monocytes differentiate into macrophages or, in some cases, into dendritic cells (DCs). Like neutrophils, macrophages phagocytose periodontal pathogens, and furthermore orchestrate wound repair by functionally coordinating innate and adaptive immune responses. Macrophages recognize oral microbes through surface Toll-like receptors (TLRs) (Muthukuru et al., 2005), which activate pro-inflammatory cytokines, chemokines, and microbicidal molecules. Thus, classically defined innate responses mediate multiple outcomes in chronically infected oral tissue.
In addition to the role cellular mechanisms play in periodontitis in lean individuals, molecular mechanisms also determine the outcome of oral infection. TLRs regulate oral inflammation in part through a chronic infection-associated mechanism termed 'endotoxin tolerance'. Endotoxin tolerance is defined as a severely blunted cytokine response to a secondary dose of endotoxin that follows closely on the heels of initial endotoxin challenge. Interestingly, $P$. gingivalis LPS, a TLR2-angonist, induces macrophage tolerance (Muthukuru et al., 2005), perhaps to facilitate $P$. gingivalis evasion from the immune system. However, new work surprisingly concludes that $P$. gingivalis exploits TLR2 in Escherichia coli LPS-tolerized macrophages to induce excessive production of $\mathrm{TNF} \alpha$, thus driving oral bone loss (Papadopoulos et al., 2013). Although a parallel role for endotoxin tolerance or the ability of $P$. gingivalis LPS to ignore $E$. coli LPS-induced tolerance has not been described in obesity, the demonstration that tolerance requires adiponectin (Zacharioudaki et al., 2009), an anti-inflammatory adipokine that is severely decreased in obesity, allows us to speculate that macrophage tolerance may not be critical in periodontitis in obese individuals.

Besides the innate immune responses of cells like fibroblasts and myeloid cells, multiple lines of evidence implicate lymphocytes in unresolved oral infection. B cells and their terminally differentiated offspring, plasma cells, dominate chronic periodontitis lesions (Berglundh and Donati, 2005). B cells spearhead multiple host defense mechanisms, including the production of antibodies that identify and bind to antigen in a highly specific "lock and key" mechanism. B-cell IgG has been proposed to protect the host from periodontopathogen infection, consistent with the finding of an inverse correlation between antipathogen antibodies and disease severity (Hall et al., 2012). However, polyclonal antibodies to periodontopathic bacteria are often non-specific and/or of low avidity, and thus may be ineffective in clearing the infection (Tew et al., 1989). Moreover, the existence of a destructive autoantibody has been proposed to contribute to periodontitis (De-Gennaro et al., 2006), which directly contradicts the protective antibody hypothesis outlined above. Related studies also showed that B cell-expressed IgD contributes to alveolar bone loss following $P$. gingivalis infection (Baker et al., 2009). However, the exact role of IgD and the specific mechanism underlying IgD function in bone loss are not known. Given the context of contradictory outcomes outlined above, the overall role of antibodies in periodontitis remains unclear. Because the role of antibodies in T2D is similarly controversial (Winer et al., 2011; Nikolajczyk et al., 2012), a definitive understanding of the role antibodies play in both diseases will require more focused approaches, such as antibodyspecific knock-out mice. In addition to antibodies, B cells from periodontitis patients also secrete a pro-inflammatory cytokine profile that is similar to the profile expressed by B cells from individuals with T2D (Nikolajczyk et al., 2012). The role for B-cell cytokines in oral pathogenesis remains to be rigorously established, in part because other cell types also secrete B-cell cytokines. A final function of B cells in periodontitis is based on demonstrations that B cells are major, and in some cases functionally unique, sources of pro-osteoclastogenic RANKL (Onal et al., 2012). RANKL expression increases in animals with 
obesity-associated T2D (Cao et al., 2010), raising the possibility that B-cell RANKL may potentiate T2D-associated periodontitis.

The second major lymphocyte subset, $\mathrm{T}$ cells, constitutes the second most numerous immune cell in chronic periodontal lesions, outnumbered only by B cells (Berglundh and Donati, 2005). The $\mathrm{CD}^{+}{ }^{+} \mathrm{T}$-cell subset plays a significant role in periodontitis, as evidenced by the demonstration that $\mathrm{CD}^{+}$(but not $\mathrm{CD}^{+}$) T-cell deficiency decreases $P$. gingivalis-induced oral bone loss (Baker et al., 2002). Recent studies also showed that $P$. gingivalis induces RANKL expression on $\mathrm{CD}^{+} \mathrm{T}$ cells (Belibasakis et al., 2011), and that RANKL neutralization can block the bone destruction presumably induced by these cells (Lin et al., 2011). Although analysis of these data suggests that CD4+ T-cell RANKL is critical for periodontitis, definitive experiments need to be completed to determine whether T-cell RANKL alone or in combination with B-cell RANKL plays major roles in pathogen-induced oral bone loss, and whether obesity similarly increases RANKL from multiple cellular sources.

Of the multiple types of $\mathrm{CD}^{+} \mathrm{T}$ cells (Th1, Th2, Th17, Tregs), Th2 cells are thought to dominate over an initial Th1 response in progressive periodontitis (Yamamoto et al., 1997), perhaps reminiscent of Th2-dominated immune hyper-reactivity in asthma. A more recently appreciated $\mathrm{CD}^{+} \mathrm{T}$-cell subset, the anti-inflammatory Tregs, is also present in the periodontal tissue (Cardoso et al., 2008) and could theoretically attenuate the severity of experimental periodontitis, regardless of Th1 vs. Th2 origins. Diseased periodontal tissues are also populated by abundant Th17 cells (Moutsopoulos et al., 2012), which express the osteoclastogenic cytokine IL-17. Interestingly, $P$. gingivalis favors Th17 differentiation not only by triggering antigenpresenting cells to produce Th17-supporting cytokines, but also by differentially degrading cytokines that promote Th1 (over Th17) differentiation (Moutsopoulos et al., 2012). The speculation that $P$. gingivalis "engineers" a Th17 environment over a developmentally related Th1 milieu is inconsistent with the idea that Th1s characterize non-progressive lesions (Yamamoto et al., 1997), and suggests instead that Th17s represent the lesser of 2 evils for the bacteria. Overall, analysis of the data provides controversy yet little concrete evidence regarding the role of specific $\mathrm{CD}^{+} \mathrm{T}$-cell subsets in periodontitis.

\section{IMMUNE RESPONSES IN T2D-ASSOCIATED PERIODONTITIS}

Examinations of the immune responses that characterize T2Dassociated periodontitis have begun to query the possibility that dysregulated immune responses potentiate periodontitis in T2D. T2D-associated changes in innate responses of gingival epithelial cells, fibroblasts, and endothelial cells include increased expression of receptors of advanced glycation end-products, or RAGEs (Katz et al., 2005), which broadly activate inflammation and thus may predispose toward periodontitis (Amir et al., 2011). The importance of RAGE/AGE interaction in T2Dassociated periodontitis is experimentally supported by studies in which systemic administration of soluble RAGE prevented periodontal disease progression in a lean type 1 diabetes mouse model that recapitulates the hyperglycemia of obese/T2D mice (Lalla et al., 2000). Clinical and animal studies focused on cellular mechanisms also provide evidence for aberrant immune cell function - for example, enhanced neutrophil superoxide production - in T2D-associated periodontitis (Karima et al., 2005). Like altered RAGE/AGE outcomes, altered neutrophil function may stem, at least in part, from T2D-associated hyperglycemia, which primes mouse gingival neutrophils for marginalization and superoxide production (Gyurko et al., 2006).

In contrast to the rich literature implicating macrophages in obesity-associated inflammation and T2D, outlined above, there is, perhaps surprisingly, no direct evidence of a role for monocytes/ macrophages in T2D-associated periodontitis in humans. However, one animal study showed that macrophages in obese mice respond to $P$. gingivalis with lower inflammatory cytokine production compared with macrophages from lean hosts (Amar et al., 2007). Importantly, these mice differed from standard diet-induced obese mice, as evidenced by the absence of hyperglycemia. This unexpected decrease in macrophage response is consistent with the idea that some immune system functions may be blunted by obesity/T2D and is consistent with the dysregulated TLR expression and significantly reduced signal transduction in peritoneal macrophages responding to $P$. gingivalis in work from the same group (Zelkha et al., 2010). Because both saturated fatty acid and LPS could require TLRs to activate cells, it is possible that chronic exposure to saturated fatty acids in obesity with confounding metabolic disease induces macrophage tolerance in vitro (Zelkha et al., 2010). However, recent studies showed that saturated fatty acids instead amplified inflammatory signaling triggered by LPS in macrophages in vitro (Jin et al., 2013), directly contradicting the tolerance hypothesis in obesity/T2D-associated periodontitis. Analysis of macrophages in a more standard in vivo mouse model (discussed below) may clarify these seemingly disparate results. Finally, one underappreciated possibility to explain how altered immune responses support both obesity-associated inflammation and oral pathogen infection is work showing that, surprisingly, abnormal cytokine persistence alone can prevent pathogen clearance (Wilson et al., 2013).

Evidence for a role of altered lymphocyte function in diabetic patients with periodontitis is similarly limited. Preliminary evidence for an association between glycemic control and IFN$\gamma /$ IL-17 concentrations in gingival crevicular fluid from periodontitis patients with diabetes (Santos et al., 2010; Ribeiro et al., 2011) suggests that Th1/Th17 might link periodontitis and T2D. A recent immunohistological study (Duarte et al., 2011) also provides evidence for elevated percentages of Th17 and Treg cells in patients with T2D plus periodontitis as compared with patients with periodontitis alone. Similarly, few studies have tested roles for B cells in T2D-associated periodontitis. Preliminary work showed no significant difference in B-cell infiltrate in gingival tissue in T2D- $v s$. non-diabetes-associated periodontitis (Duarte et al., 2011). However, given the predominance of $\mathrm{B}$ cells in periodontitis lesions, our demonstration that $\mathrm{B}$ cells from obese individuals with $\mathrm{T} 2 \mathrm{D}$ express a proinflammatory cytokine profile similar to that of B cells from lean individuals with periodontitis (Nikolajczyk et al., 2012; DeFuria et al., 2013) leads us to speculate that the phenotypic changes in B cells in obesity may have the unexpected consequence of promoting periodontal disease. Overall, the role of 


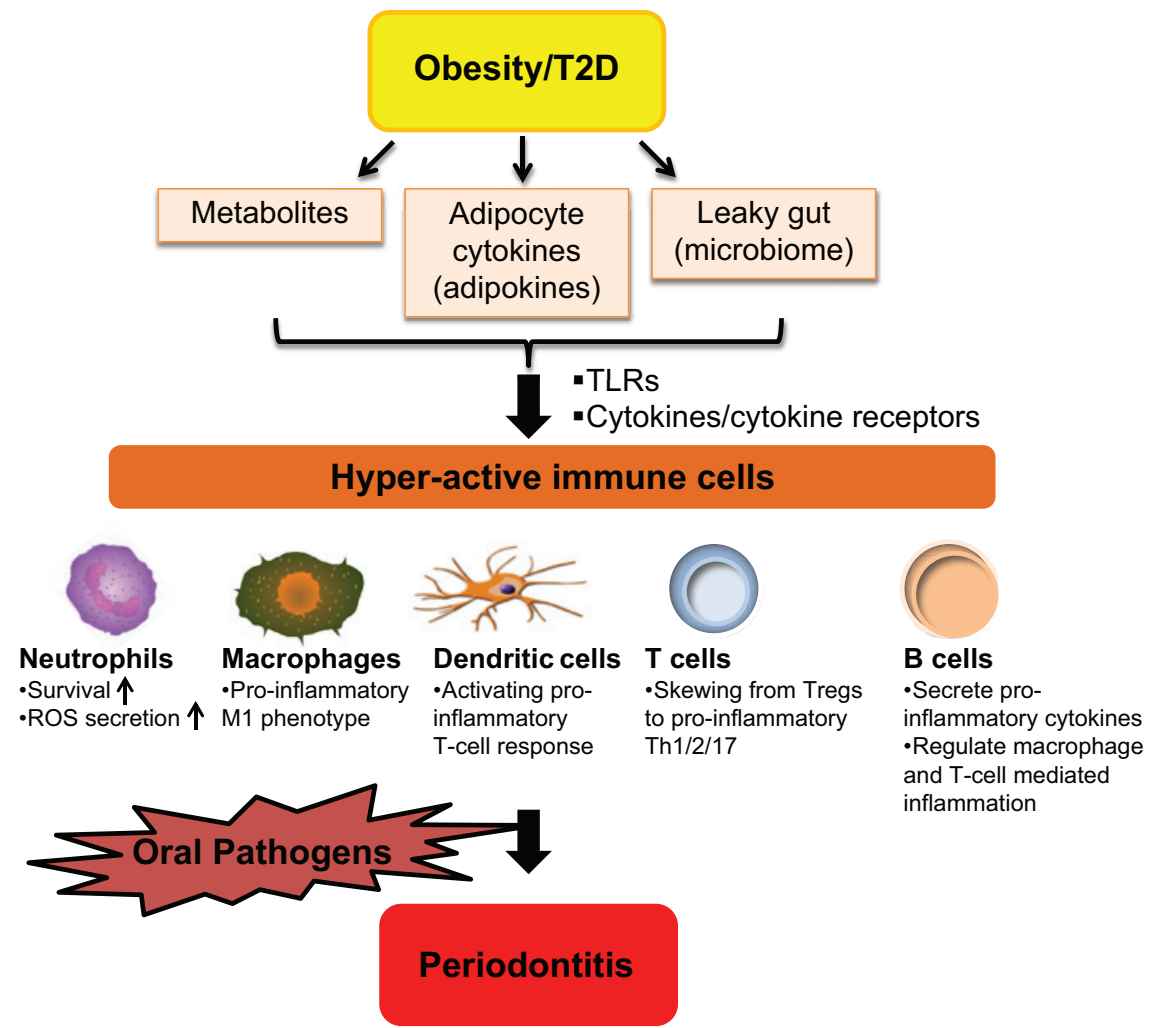

Figure 2. Working hypothesis of the link between obesity-associated type 2 diabetes (T2D) and periodontitis. Obesity-associated T2D results in increased pro-inflammatory metabolites such as free fatty acids and glucose as well as endotoxin from increased gut permeability. Obesity also results in higher concentrations of adipocyte cytokines, as well as the altered intestinal microbiota, all of which trigger surface signaling molecules including Toll-like receptors (TLRs) and cytokine receptors. These receptors activate a wide array of immunological cells types, including most immune system cells as shown, and structural cells with innate immune responses such as adipocytes, gingival epithelial cells, and fibroblasts (not shown). Thus, obesity/T2D primes immune mechanisms for an inappropriately robust response to oral pathogens that results in periodontal inflammation and exaggerated tissue destruction.

interpreted with special caution because of the appreciation that $\mathrm{T}$ cells require leptin for normal development and function (Lord et al., 1998; Palmer et al., 2006). Furthermore, current animal models of obesity are generally characterized by insulin resistance; therefore, these models cannot address periodontal diseases in MHO or in obese subjects who are creeping toward T2D yet maintain some insulin sensitivity and beta cell function. Despite the limitations of the available animal models, the diet-induced obese (DIO) model, in which animals become both obese and insulin-resistant in response to a high-fat diet (Nilsson et al., 2012), may be an important tool for understanding periodontitis potentiated by obesity-associated T2D. Genetic manipulations have been used in conjunction with DIO to establish the role of specific immune cell types in obesity/T2D, including but not limited to B cell-deficient mice (Winer et al., 2011; DeFuria et al., 2013) and $\mathrm{CD}^{+} \mathrm{T}$ cell-deficient mice (Nishimura et al., 2009). In theory, DIO studies in such mice could be combined with properly timed oral pathogen inoculation to identify mechanisms that promote periodontitis in obesity/T2D (proof of principle) and/or produce outcomes that can be translated to humans (e.g., phenotype of B cell-null mice can be mimicked by generally safe B-cell depletion drugs). However, caution is important in identifying genetically altered mice that are appropriate for DIO studies. Genetic

lymphocytes in periodontal pathogenesis in T2D is worthy of more comprehensive investigation in both animal models and humans, the latter of which will require logistically challenging collaborations between endocrinology and periodontology clinics to ensure thorough characterization of both metabolic and periodontal status in study participants.

\section{ANIMAL MODELS AS TOOLS FOR MECHANISTIC STUDIES OF OBESITY/T2D-ASSOCIATED PERIODONTITIS}

Multiple models of periodontitis in animals with a combination of obesity and metabolic disease suggest that immune responses predispose obese/T2D individuals to periodontitis, but these studies have limitations. For example, the monogenic nature of some animal models may not accurately recapitulate human pathogenesis, since only a few cases of human obesity/T2D are due to gene mutations (Nilsson et al., 2012). The use of genetically obese ob/ ob or $\mathrm{db} / \mathrm{db}$ mice, which contain a natural mutation that inactivates leptin or the leptin receptor, respectively, must be manipulation may lead to divergence of body weight, an important determinant of inflammation and insulin resistance. In the vast majority of cases, a heavier mouse is more inflamed and insulin-resistant, thus likely more susceptible to periodontitis. In such cases, genetic manipulation probably plays a dominant role in feeding behavior or basal metabolism, which could make results from adding periodontitis to such models tricky to interpret.

An additional consideration for the use of animal models to interpret human disease pathogenesis in obesity-associated periodontitis is the method used to induce periodontitis. Limited numbers of studies have used obese/T2D animals to understand periodontitis in the context of obesity/T2D, with periodontitis initiated by either mechanical methods (i.e., ligature) (Pacios et al., 2012) or by systemic $P$. gingivalis infection (Amar et al., 2007). Despite the insights into obesity/T2D-associated periodontitis reported from these studies, the acute traumatic nature of ligature-induced periodontitis (which mechanistically contrasts with chronic human periodontitis) or the non-physiological route of $P$. gingivalis entry into the infection site (i.e., by venous injection) calls for additional work with more physiological approaches to the understanding of combinatorial disease. 


\section{SUMMARY AND FUTURE DIRECTIONS}

The establishment of an association between obesity and periodontitis is beginning to shed light on concrete targets for breaking the feed-forward loops among obesity, T2D, and periodontitis and to perhaps stop disease before the dangerous transition to T2D. Currently available work on immune cell function in T2D-associated periodontitis supports an overall hyper-responsive state that reinforces chronic oral inflammation (Fig. 2). Immune cells, especially the $\mathrm{B}$ and $\mathrm{T}$ cells that dominate periodontitis lesions, are strongly implicated in obesityassociated periodontitis, but require further examination. These and other cell types, including macrophages and neutrophils, will be fruitful areas for detailed investigation.

Analysis of current data suggests several lines of inquiry that will identify targets for interrupting the interaction between obesity and periodontitis, regardless of metabolic status. Foremost, information on the histopathology of periodontal lesions in wellcharacterized obese individuals, including MHO persons, is incomplete, since many clinical studies have not taken into account and/or described the metabolic status of oral sample donors. Baseline studies of cellular infiltrates are critical for interpretation of, for example, published transcriptional profiling data from gingiva. Second, researchers must take into account differences between humans and experimental animals (mainly rodents) with regard to immune system function and metabolism. In contrast to the uncoupling of metabolic status from obesity in $\mathrm{MHO}$ individuals, animal models almost always suffer from simultaneous obesity and metabolic disease. Therefore, studies of $\mathrm{db} / \mathrm{db}$ mice in the calvarial model of periodontitis, which revealed that T2D causes impaired healing and suppression of bone formation in response to $P$. gingivalis (He et al., 2004), may be irrelevant to MHO people. Similarly, ligature-induced periodontitis in Zucker diabetic fatty rats showed that a T2D-like condition increases the intensity and duration of inflammatory infiltrate as well as bone loss (Liu et al., 2006), but the outcomes under MHO conditions were not tested.

Epidemiological and experimental studies link periodontitis with obesity-associated T2D, but the precise mechanisms underlying the interactions among obesity, metabolic health, and periodontitis remain elusive. A more thorough characterization of obesity-associated periodontitis in rodent models by a combination of oral assessment and whole-body metabolic measures will be required to complement tissue analysis of wellcharacterized individuals, and to identify mechanisms by which periodontitis may predispose to metabolic imbalance, a "holy grail" of periodontal research. Overall, studies of immune effectors using the most relevant animal model are urged to identify biologically active drugs that effectively break the cycle between obesity-associated T2D and periodontitis, to simultaneously improve multiple measures of health.

\section{ACKNOWLEDGMENTS}

This work was supported by the National Institutes of Health (grants R21DK089270, R21DE021154, NIH R56 DK096525). The authors declare no potential conflicts of interest with respect to the authorship and/or publication of this article.

\section{REFERENCES}

Amar S, Zhou Q, Shaik-Dasthagirisaheb Y, Leeman S (2007). Diet-induced obesity in mice causes changes in immune responses and bone loss manifested by bacterial challenge. Proc Natl Acad Sci USA 104:2046620471.

Amir J, Waite M, Tobler J, Catalfamo DL, Koutouzis T, Katz J, et al. (2011). The role of hyperglycemia in mechanisms of exacerbated inflammatory responses within the oral cavity. Cell Immunol 272:45-52.

Baker PJ, Howe L, Garneau J, Roopenian DC (2002). T cell knockout mice have diminished alveolar bone loss after oral infection with Porphyromonas gingivalis. FEMS Immunol Med Microbiol 34:45-50.

Baker PJ, Boutaugh NR, Tiffany M, Roopenian DC (2009). B cell IgD deletion prevents alveolar bone loss following murine oral infection. Interdiscip Perspect Infect Dis 2009:864359.

Belibasakis GN, Reddi D, Bostanci N (2011). Porphyromonas gingivalis induces RANKL in T-cells. Inflammation 34:133-138.

Berglundh T, Donati M (2005). Aspects of adaptive host response in periodontitis. J Clin Periodontol 32(Suppl 6):87-107.

Bertola A, Ciucci T, Rousseau D, Bourlier V, Duffaut C, Bonnafous S, et al. (2012). Identification of adipose tissue dendritic cells correlated with obesity-associated insulin-resistance and inducing Th17 responses in mice and patients. Diabetes 61:2238-2247.

Bosshardt DD, Lang NP (2005). The junctional epithelium: from health to disease. J Dent Res 84:9-20.

Cao JJ, Sun L, Gao H (2010). Diet-induced obesity alters bone remodeling leading to decreased femoral trabecular bone mass in mice. Ann NY Acad Sci 1192:292-297.

Cardoso CR, Garlet GP, Moreira AP, Junior WM, Rossi MA, Silva JS (2008). Characterization of CD4+CD25+ natural regulatory $\mathrm{T}$ cells in the inflammatory infiltrate of human chronic periodontitis. J Leukoc Biol 84:311-318.

Chawla A, Nguyen KD, Goh YP (2011). Macrophage-mediated inflammation in metabolic disease. Nat Rev Immunol 11:738-749.

De-Gennaro LA, Lopes JD, Mariano M (2006). Autoantibodies directed to extracellular matrix components in patients with different clinical forms of periodontitis. J Periodontol 77:2025-2030.

DeFuria J, Belkina AC, Jagannathan-Bogdan M, Snyder-Cappione J, Carr JD, Nersesova YR, et al. (2013). B cells promote inflammation in obesity and type 2 diabetes through regulation of T-cell function and an inflammatory cytokine profile. Proc Natl Acad Sci USA 110:51335138.

Denis GV, Obin MS (2013). 'Metabolically healthy obesity': origins and implications. Mol Aspects Med 34:59-70.

Dias IH, Matthews JB, Chapple IL, Wright HJ, Dunston CR, Griffiths HR (2011). Activation of the neutrophil respiratory burst by plasma from periodontitis patients is mediated by pro-inflammatory cytokines. J Clin Periodontol 38:1-7.

Duarte PM, Santos VR, Dos Santos FA, de Lima Pereira SA, Rodrigues DB, Napimoga MH (2011). Role of smoking and type 2 diabetes in the immunobalance of advanced chronic periodontitis. $J$ Periodontol $82: 429-438$.

Duffaut C, Galitzky J, Lafontan M, Bouloumié A (2009). Unexpected trafficking of immune cells within the adipose tissue during the onset of obesity. Biochem Biophys Res Commun 384:482-485.

Elgazar-Carmon V, Rudich A, Hadad N, Levy R (2008). Neutrophils transiently infiltrate intra-abdominal fat early in the course of high-fat feeding. J Lipid Res 49:1894-1903.

Feuerer M, Herrero L, Cipolletta D, Naaz A, Wong J, Nayer A, et al. (2009). Lean, but not obese, fat is enriched for a unique population of regulatory $\mathrm{T}$ cells that affect metabolic parameters. Nat Med 15:930-939.

Gorman A, Kaye EK, Apovian C, Fung TT, Nunn M, Garcia RI (2012). Overweight and obesity predict time to periodontal disease progression in men. J Clin Periodontol 39:107-114.

Gyurko R, Siqueira CC, Caldon N, Gao L, Kantarci A, Van Dyke TE (2006). Chronic hyperglycemia predisposes to exaggerated inflammatory response and leukocyte dysfunction in Akita mice. J Immunol 177:7250-7256.

Hajishengallis E, Hajishengallis G (2014). Neutrophil homeostasis and periodontal health in children and adults. J Dent Res 93:231-237. 
Hall LM, Dunford RG, Genco RJ, Sharma A (2012). Levels of serum immunoglobulin $\mathrm{G}$ specific to bacterial surface protein A of Tannerella forsythia are related to periodontal status. J Periodontol 83:228-234.

He H, Liu R, Desta T, Leone C, Gerstenfeld LC, Graves DT (2004). Diabetes causes decreased osteoclastogenesis, reduced bone formation, and enhanced apoptosis of osteoblastic cells in bacteria stimulated bone loss. Endocrinology 145:447-452.

Jimenez M, Hu FB, Marino M, Li Y, Joshipura KJ (2012). Prospective associations between measures of adiposity and periodontal disease. Obesity 20:1718-1725.

Jin J, Zhang X, Lu Z, Perry DM, Li Y, Hannun YA, et al. (2013). Acid sphingomyelinase plays a key role in palmitic acid-amplified inflammatory signaling triggered by lipopolysaccharide at low concentration in macrophages. Am J Physiol Endocrinol Metab 305:E853-E867.

Karima M, Kantarci A, Ohira T, Hasturk H, Jones VL, Nam BH, et al. (2005). Enhanced superoxide release and elevated protein kinase C activity in neutrophils from diabetic patients: association with periodontitis. J Leukoc Biol 78:862-870.

Katz J, Bhattacharyya I, Farkhondeh-Kish F, Perez FM, Caudle RM, Heft MW (2005). Expression of the receptor of advanced glycation end products in gingival tissues of type 2 diabetes patients with chronic periodontal disease: a study utilizing immunohistochemistry and RT-PCR. J Clin Periodontol 32:40-44.

Kopelman P (2007). Health risks associated with overweight and obesity. Obes Rev 8 (Suppl 1):13-17.

Lakschevitz FS, Aboodi GM, Glogauer M (2013). Oral neutrophil transcriptome changes result in a pro-survival phenotype in periodontal diseases. Plos One 8:e68983.

Lalla E, Lamster IB, Feit M, Huang L, Spessot A, Qu W, et al. (2000). Blockade of RAGE suppresses periodontitis-associated bone loss in diabetic mice. $J$ Clin Invest 105:1117-1124.

Lin X, Han X, Kawai T, Taubman MA (2011). Antibody to receptor activator of NF-kappaB ligand ameliorates $\mathrm{T}$ cell-mediated periodontal bone resorption. Infect Immun 79:911-917.

Liu R, Bal HS, Desta T, Krothapalli N, Alyassi M, Luan Q, et al. (2006). Diabetes enhances periodontal bone loss through enhanced resorption and diminished bone formation. $J$ Den Res 85:510-514.

Lord GM, Matarese G, Howard JK, Baker RJ, Bloom SR, Lechler RI (1998). Leptin modulates the T-cell immune response and reverses starvation-induced immunosuppression. Nature 394:897-901.

Lumeng CN, Bodzin JL, Saltiel AR (2007). Obesity induces a phenotypic switch in adipose tissue macrophage polarization. J Clin Invest 117:175184.

Miyauchi M, Sato S, Kitagawa S, Hiraoka M, Kudo Y, Ogawa I, et al. (2001). Cytokine expression in rat molar gingival periodontal tissues after topical application of lipopolysaccharide. Histochem Cell Biol 116:57-62.

Moutsopoulos NM, Kling HM, Angelov N, Jin W, Palmer RJ, Nares S, et al. (2012). Porphyromonas gingivalis promotes Th17 inducing pathways in chronic periodontitis. J Autoimmun 39:294-303.

Muthukuru M, Jotwani R, Cutler CW (2005). Oral mucosal endotoxin tolerance induction in chronic periodontitis. Infect Immun 73:687-694.

Nikolajczyk BS, Jagannathan-Bogdan M, Denis GV (2012). The outliers become a stampede as immunometabolism reaches a tipping point. Immunol Rev 249:253-275.

Nilsson C, Raun K, Yan FF, Larsen MO, Tang-Christensen M (2012). Laboratory animals as surrogate models of human obesity. Acta Pharmacol Sin 33:173-181.

Nishimura S, Manabe I, Nagasaki M, Eto K, Yamashita H, Ohsugi M, et al. (2009). CD8(+) effector T cells contribute to macrophage recruitment and adipose tissue inflammation in obesity. Nat Med 15:914-920

Oh DY, Morinaga H, Talukdar S, Bae EJ, Olefsky JM (2012). Increased macrophage migration into adipose tissue in obese mice. Diabetes 61:346-354.
Onal M, Xiong J, Chen X, Thostenson JD, Almeida M, Manolagas SC, et al. (2012). Receptor activator of nuclear factor kappaB ligand (RANKL) protein expression by B lymphocytes contributes to ovariectomyinduced bone loss. J Biol Chem 287:29851-29860.

Pacios S, Kang J, Galicia J, Gluck K, Patel H, Ovaydi-Mandel A, et al. (2012). Diabetes aggravates periodontitis by limiting repair through enhanced inflammation. FASEB $J$ 26:1423-1430.

Palmer G, Aurrand-Lions M, Contassot E, Talabot-Ayer D, Ducrest-Gay D, Vesin C, et al. (2006). Indirect effects of leptin receptor deficiency on lymphocyte populations and immune response in $\mathrm{db} / \mathrm{db}$ mice. J Immunol 177:2899-2907.

Papadopoulos G, Weinberg EO, Massari P, Gibson FC 3rd, Wetzler LM, Morgan EF, et al. (2013). Macrophage-specific TLR2 signaling mediates pathogen-induced TNF-dependent inflammatory oral bone loss. J Immunol 190:1148-1157.

Preshaw PM, Alba AL, Herrera D, Jepsen S, Konstantinidis A, Makrilakis $\mathrm{K}$, et al. (2012). Periodontitis and diabetes: a two-way relationship. Diabetologia 55:21-31.

Ribeiro FV, de Mendonca AC, Santos VR, Bastos MF, Figueiredo LC, Duarte PM (2011). Cytokines and bone-related factors in systemically healthy patients with chronic periodontitis and patients with type 2 diabetes and chronic periodontitis. $J$ Periodontol 82:1187-1196.

Rocha VZ, Folco EJ, Sukhova G, Shimizu K, Gotsman I, Vernon AH, et al. (2008). Interferon-gamma, a Th1 cytokine, regulates fat inflammation: a role for adaptive immunity in obesity. Circ Res 103:467-476.

Saberi M, Woods NB, de Luca C, Schenk S, Lu JC, Bandyopadhyay G, et al. (2009). Hematopoietic cell-specific deletion of Toll-like receptor 4 ameliorates hepatic and adipose tissue insulin resistance in highfat-fed mice. Cell Metab 10:419-429.

Santos VR, Ribeiro FV, Lima JA, Napimoga MH, Bastos MF, Duarte PM (2010). Cytokine levels in sites of chronic periodontitis of poorly controlled and well-controlled type 2 diabetic subjects. J Clin Periodontol 37:1049-1058.

Stefanovic-Racic M, Yang X, Turner MS, Mantell BS, Stolz DB, Sumpter TL, et al. (2012). Dendritic cells promote macrophage infiltration and comprise a substantial proportion of obesity-associated increases in CD11c+ cells in adipose tissue and liver. Diabetes 61:2330-2339.

Suvan J, D'Aiuto F, Moles DR, Petrie A, Donos N (2011). Association between overweight/obesity and periodontitis in adults. A systematic review. Obes Rev 12:e381-e404.

Swinburn BA, Sacks G, Hall KD, McPherson K, Finegood DT, Moodie ML, et al. (2011). The global obesity pandemic: shaped by global drivers and local environments. Lancet 378:804-814.

Tew J, Engel D, Mangan D (1989). Polyclonal B-cell activation in periodontitis. J Periodontal Res 24:225-241.

Wilson EB, Yamada DH, Elsaesser H, Herskovitz J, Deng J, Cheng G, et al. (2013). Blockade of chronic type I interferon signaling to control persistent LCMV infection. Science 340:202-207.

Winer DA, Winer S, Shen L, Wadia PP, Yantha J, Paltser G, et al. (2011). $\mathrm{B}$ cells promote insulin resistance through modulation of $\mathrm{T}$ cells and production of pathogenic IgG antibodies. Nat Med 17:610-617.

Winer S, Chan Y, Paltser G, Truong D, Tsui H, Bahrami J, et al. (2009). Normalization of obesity-associated insulin resistance through immunotherapy. Nat Med 15:921-929.

Yamamoto M, Fujihashi K, Hiroi T, McGhee JR, Van Dyke TE, Kiyono H (1997). Molecular and cellular mechanisms for periodontal diseases: role of Th1 and Th2 type cytokines in induction of mucosal inflammation. J Periodontal Res 32(1Pt 2):115-119.

Zacharioudaki V, Androulidaki A, Arranz A, Vrentzos G, Margioris AN, Tsatsanis C (2009). Adiponectin promotes endotoxin tolerance in macrophages by inducing IRAK-M expression. J Immunol 182:6444-6451.

Zelkha SA, Freilich RW, Amar S (2010). Periodontal innate immune mechanisms relevant to atherosclerosis and obesity. Periodontol 2000 54:207-221. 